\title{
Analysis of the Influence of Parallelism Error on the Meshing Performance of Circular-Arc-Tooth-Trace Cylindrical Gears
}

\author{
Tang Rui*, Ling Yun \\ Aviation Engineering Institute, Civil Aviation Flight University of China, Guanghan, China \\ Email address: \\ pzhutr@163.com (Tang Rui) \\ ${ }^{*}$ Corresponding author \\ To cite this article: \\ Tang Rui, Ling Yun. Analysis of the Influence of Parallelism Error on the Meshing Performance of Circular-Arc-Tooth-Trace Cylindrical \\ Gears. International Journal of Science and Qualitative Analysis. Vol. 5, No. 1, 2019, pp. 1-5. doi: 10.11648/j.ijsqa.20190501.11
}

Received: April 21, 2019; Accepted: June 2, 2019; Published: June 13, 2019

\begin{abstract}
In this paper, two types of point contact and line contact of spiral cylindrical gear transmission pair are studied and analyzed. The influences of the axis parallelism error and the center distance error on the contact area are analyzed. The numerical example shows that the line-contact transmission pair has larger contact area if the installation error is not considered. By using MATLAB software and simulation example, the influence of axis parallelism on contact area and meshing performance of spiral cylindrical gears under different error conditions are obtained.
\end{abstract}

Keywords: Circular-Arc-Tooth-Trace Cylindrical Gear, Parallelism Error, Meshing Performance

\section{Introduction}

The gear is extensively used in various mechanical and equipment applications as important basic component. [1] Its performance largely determines the capability of equipment. [2] Even more, the applications to aerospace and defense technology put forward higher requirement to transmission, such as high reliability, lightweight and lower noise. Therefore, it shows quite vital theoretical significance and practical engineering value to develop high-performance transmission component deeply. [3] Mechanical basic parts largely determine the performance of mechanical equipment, and have a great impact on the development of manufacturing. Gear transmission is one of the most widely used forms of transmission and is a very important part of mechanical equipment. The meshing characteristics of the gear transmission directly affect the performance of the mechanical equipment. The development of the high-performance meshing pair is an eternal theme of academic research and the development of the gear industry [4]. As a new type of gear, the arc-toothed cylindrical gear has the characteristics of good meshing performance, long contact line, large coincidence degree, no axial component, large bearing capacity, high transmission efficiency and stable transmission. Its comprehensive performance is better. Ordinary cylindrical gears have a good application prospect [5].

\section{Meshing Analysis of Circular-Arc-Tooth-Trace Cylindrical Gear}

\subsection{Contact Form}

The arc milling gear is manufactured by the improved milling method [6]. The machining method is easy to realize, the machine tool design is simple, the precision of the transmission pair is easy to guarantee, and the production efficiency is high. There are two ideal meshing conditions for this type of gear transmission pair, which are point contact and line contact. The arc-toothed cylindrical gear transmission proposed in this paper has two working modes. Firstly, the driving gear and the driven gear are all milled by the double-edged (tooth) tool of the large cutter, and the convex tooth surface forming radius of the gear teeth less than the concave tooth surface forming radius. In the gear transmission, the concave and convex moments of the active and driven gears are in point contact, and the contact area is an ellipse during normal operation, and the motion and power are 
transmitted through the area. Secondly, the active gear and the passive gear are machined by double-edged gear cutter respectively. When two gears are used in pairs, the instantaneous contact of gear transmission is linear contact. The contact area is a belt-shaped area through which movement and power are transmitted. In point contact, the gear is insensitive to installation error, and the lubrication film

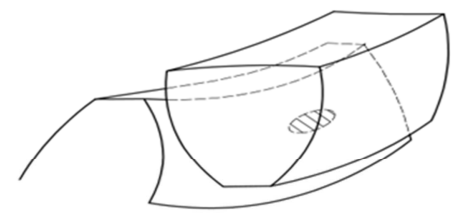

(a) Point contact is easy to form. The bearing capacity of the gear depends on the size of the contact ellipse. In line contact, gear is more sensitive to installation error than point contact, but its contact area is strip-like, its theoretical contact area is larger than point contact, and it has higher load-carrying capacity. The two contact forms are shown in Figure 1.

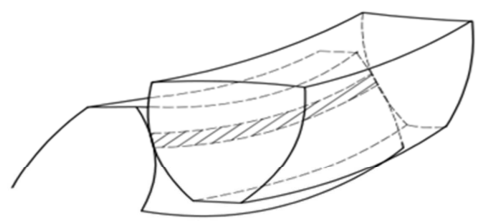

(b) Line contact

Figure 1. Contact forms of circular toothed cylindrical gear transmission pair.

\subsection{Contact Ellipse}

For the intermeshing circular toothed cylindrical gears, if the radius of the tooth forming cutter is different, the concave and convex tooth surfaces are theoretically point contact. During transmission, due to the elastic deformation of the tooth surface, the instantaneous contact of the two tooth surfaces at one point will expand from a theoretical point to an elliptical region. As shown in Figure 2, the symmetry center of the instantaneous contact ellipse coincides with the theoretical tangent contact. In fact, the flank contact marks are a series of contact ellipses, and the size of these contact ellipses will affect the transmission performance of the gear pair. The literature [7] proves that the projection of the traditional gear transmission contact area on the cutting plane is ellipse, and points out that the amount of elastic deformation of the tooth surface is related to the applied load. It is generally considered to be a given value, which can be obtained from the test data under the action of small load. The value is generally 0.00025 inch. In this paper, the contact ellipse under the action of small load is considered, so it is chosen to be $0.00632 \mathrm{~mm}$.

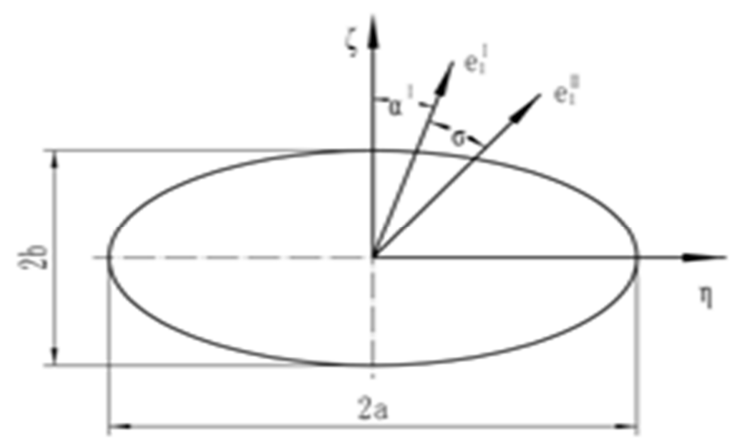

Figure 2. Contact ellipse.

According to the foregoing analysis, the contact elliptic equation of the arc-toothed cylindrical gear can be obtained as follows:

$$
A \xi^{2}+B \eta^{2}= \pm \delta
$$

The length of the ellipse can be expressed as.

$$
a=\left|\frac{\delta}{A}\right|^{1 / 2} \quad b=\left|\frac{\delta}{B}\right|^{1 / 2}
$$

Among the formulas.

$$
\begin{gathered}
A=\frac{1}{4}\left[k_{\Sigma}^{\mathrm{I}}-k_{\Sigma}^{\mathrm{II}}-\left(g_{\mathrm{I}}^{2}-2 g_{\mathrm{I}} g_{\mathrm{II}} \cos 2 \sigma+g_{\mathrm{II}}^{2}\right)^{1 / 2}\right] \\
B=\frac{1}{4}\left[k_{\Sigma}^{\mathrm{I}}-k_{\Sigma}^{\mathrm{II}}+\left(g_{\mathrm{I}}^{2}-2 g_{\mathrm{I}} g_{\mathrm{II}} \cos 2 \sigma+g_{\mathrm{II}}^{2}\right)^{1 / 2}\right] \\
k_{\Sigma}^{i}=k_{1}^{i}+k_{2}^{i}(i=\mathrm{I}, \mathrm{II}) g_{i}=k_{1}^{i}-k_{2}^{i}(i=\mathrm{I}, \mathrm{II}) \\
\cos 2 \alpha^{\mathrm{I}}=\frac{g_{\mathrm{I}}-g_{\mathrm{II}} \cos 2 \sigma}{\left(g_{\mathrm{I}}^{2}-2 g_{\mathrm{I}} g_{\mathrm{II}} \cos 2 \sigma+g_{\mathrm{II}}^{2}\right)^{1 / 2}}
\end{gathered}
$$

The elliptical area can be expressed as.

$$
S=\pi a b
$$

As the applied load increases, the value increases, which will increase the long and short halves of the contact ellipse, and the elliptical area will increase. This means that the actual tooth surface area will be larger, generally much larger than the theoretical calculation of the contact ellipse area described above. In order to facilitate the study of the influence of gear parameters on the contact ellipse, the subsequent analysis is mainly for the theoretical contact ellipse calculation.

\section{Influence of Parallelism Error on Meshing Performance of Spiral Cylindrical Gear}

In order to analyze the influence of parallelism on contact ellipse, numerical simulation was carried out by using MATLAB software. Suppose the modulus $M=4$, the number 
of teeth $\mathrm{Z1}=31$ for gear 1 , the number of teeth $\mathrm{Z} 2=69$ for gear 2 , the nominal cutter radius $\mathrm{R}=400 \mathrm{~mm}$, the pressure angle of indexing circle is 20 degrees, and the tooth width $\mathrm{B}=$ $80 \mathrm{~mm}$, the position angle of the tooth profile is 0 degree [8-9].

The arc-toothed spur gears processed by the single-edged and improved double-edged pairing and the double-edged knives have different contact conditions. The single-edged tool and the improved double-edged tool are in line contact, and the two gear axes are not parallel. At the time of load concentration, the phenomenon of load concentration is obvious, as shown in Figure. 3 (a). The gears processed by the double-edged gear tooth cutters are in point contact, and the sensitivity to the parallelism of the axis is lower than that in the line contact, as shown in Figure. 3 (b). In order to avoid the generality, the line contact is regarded as a special case of point contact, and the influence of the parallelism error of the arc-toothed cylindrical gear meshing pair can be analyzed.

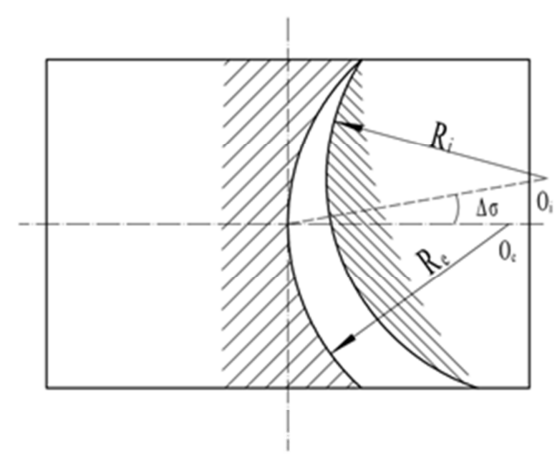

(a) Point contact

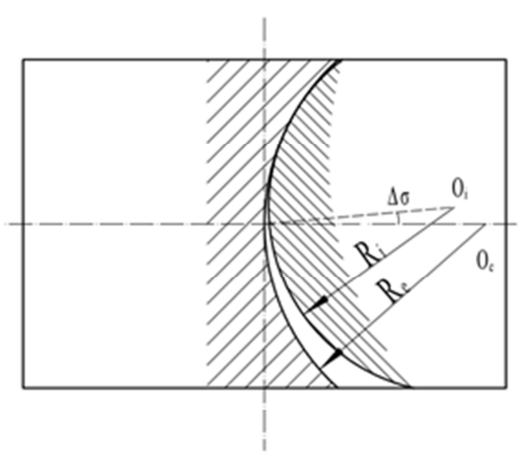

(b) Line contact

Figure 3. Effect of gear axis parallelism on meshing.

As shown in Figure 3 (b), For the sake of generality, line contact is regarded as a special case of point contact. The influence of parallelism error on the meshing pair of circular arc tooth-line cylindrical gears in point contact can be analyzed. [10]. The angle between the axes is proportional to the difference between the radius of the concave and convex toothed cutters. This shows that when the difference between the radius of the concave and convex tooth faces is small, although the tooth surface of the transmission pair gradually appears line contact, the error of the axis parallelism of the gear mounting has a greater influence on the meshing area, and vice versa. In actual use, since the manufacturing and installation errors cannot be avoided, the point contact pair has both low error sensitivity and a suitable contact area and carrying capacity, so it has better comprehensive performance.

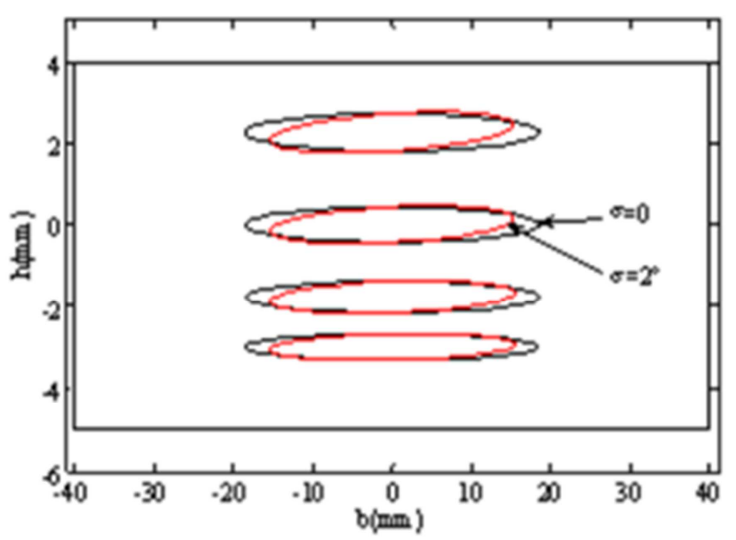

(a) The error is $2^{\circ}$

\section{Simulation Analysis}

In order to analyze the influence of parallelism on the contact ellipse, numerical simulation was performed using MATLAB software. The modulus, the number of teeth of the gear 1 , the number of teeth of the gear 2 , the nominal cutter radius, the indexing circle pressure angle, the tooth width, and the tooth profile position angle. The influence of the parallelism error on the contact area is shown in Figure 5 and Figure 6, wherein the coordinates in the tooth width direction which are indicated by the coordinates in the tooth height direction. The effect of the parallelism of the gear axis on the contact area of the gear pair of the point contact and the line contact is shown in Figure 4 and Figure 5 under different error conditions

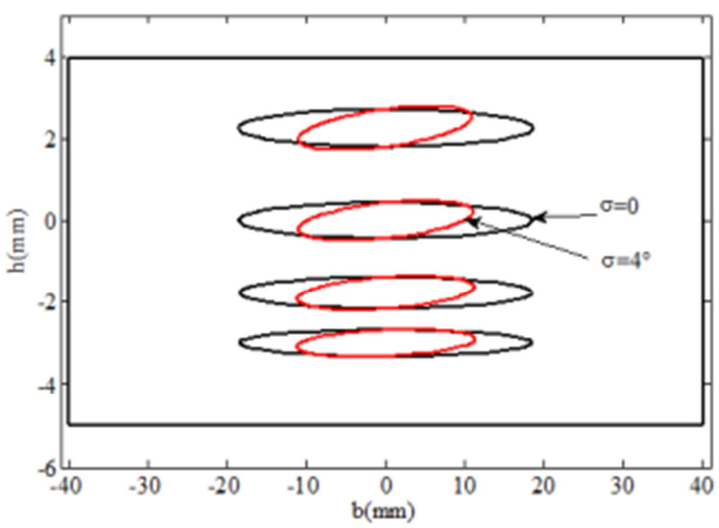

(b) The error is $4^{\circ}$

Figure 4. The influence of the parallelism of the axis on the contact area of the point contact gear pair. 


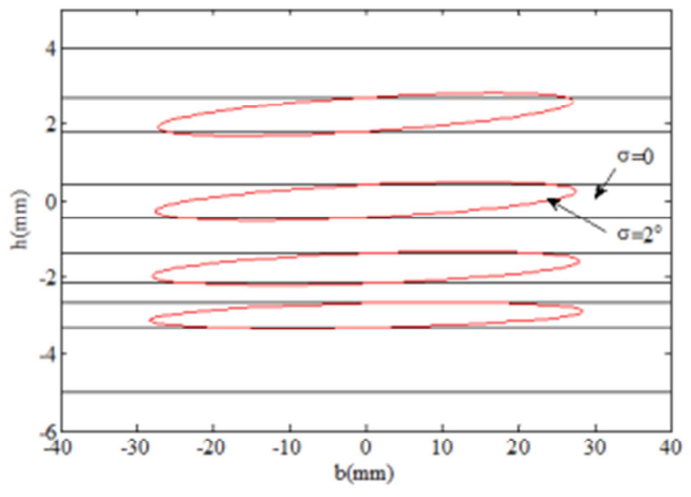

(a) The error is $2^{\circ}$

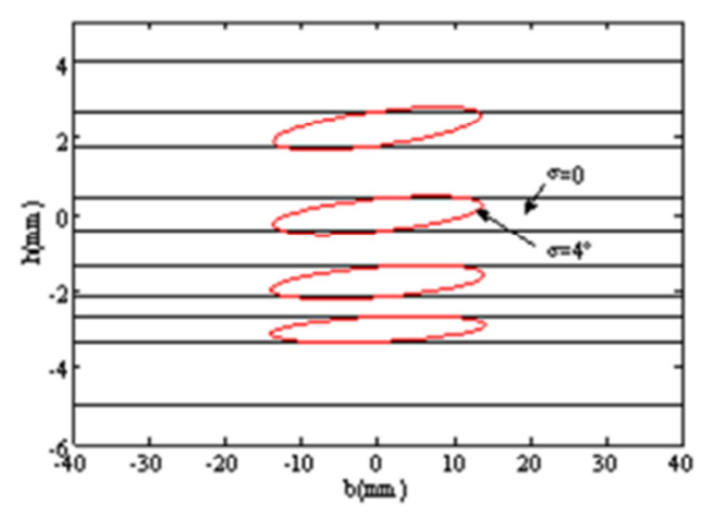

(b) The error is $4^{\circ}$

Figure 5. The influence of the parallelism of the axis on the contact area of the line contact gear pair.

As can be seen from Figure 4, the axial non-parallelism error causes the elliptical shape and size of the point contact area to change, and the larger the error, the smaller the contact area. As can be seen from Figure 5, the parallelism error causes the gear pair to become point contact from the line contact, and the contact area is changed from a strip shape to

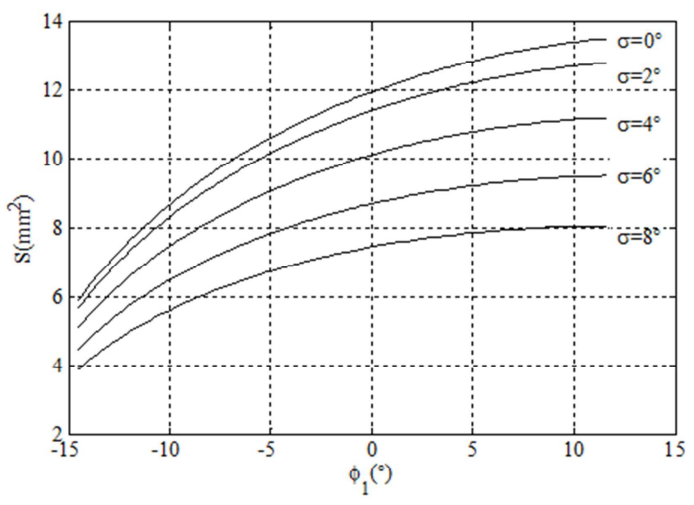

(a) Contact ellipse area an elliptical shape. By indicating the area of the contact area, indicating the ratio of the area of the contact area having the parallelism error to the area of the ideal mounting contact area, the influence of the error of the gear axis parallelism on the area of the contact area can be obtained, as shown in Figure 6 and Figure 7.

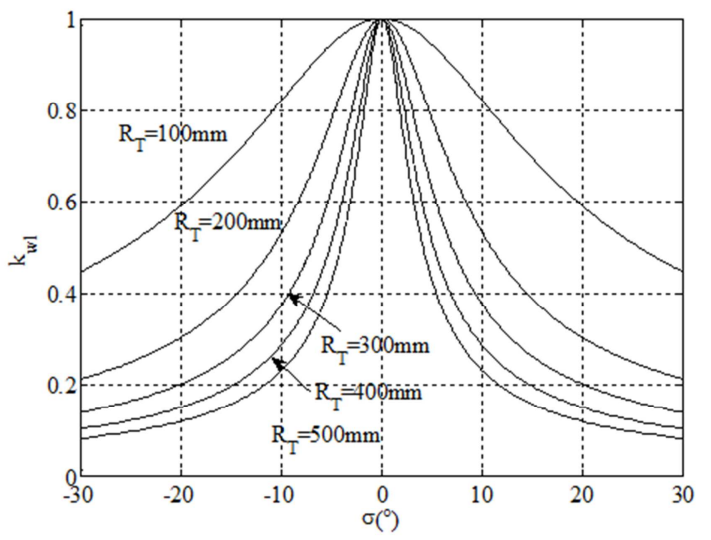

(b) Area ratio

Figure 6. The effect of the parallelism of the axis on the area of the contact ellipse (point contact).

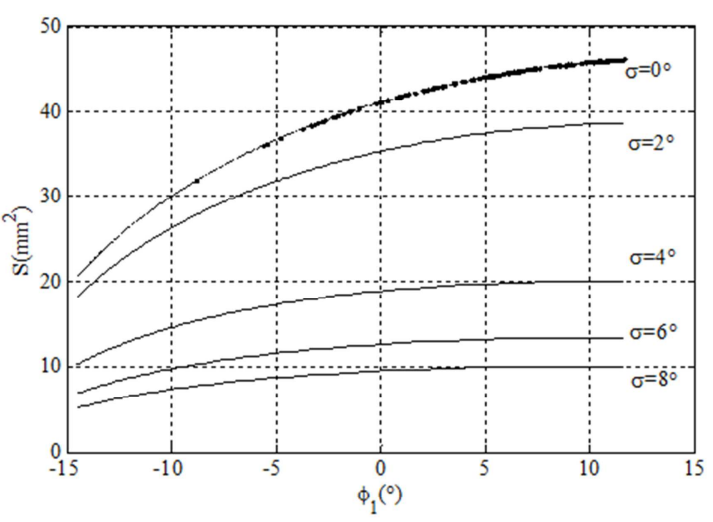

(a) Contact ellipse area

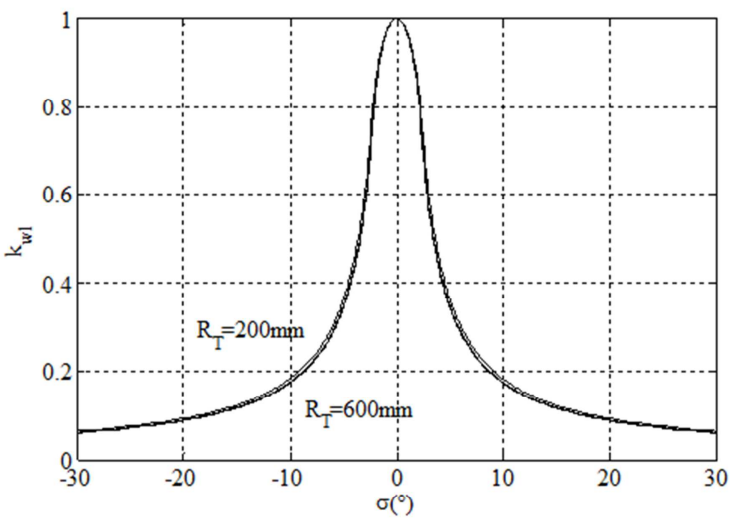

(b) Area ratio

Figure 7. Effect of the parallelism of the axis on the area of the contact area (line contact).

For point contact, the increase of the error leads to a decrease in the contact ellipse area, and the area of the meshing area is smaller than that of the tooth tip, as shown in Figure 6 (a); the increase of the nominal cutter radius 
increases the contact area. However, it is more affected by the error, as shown in Figure 6 (b). For line contact, the error increases and the contact area is greatly reduced. The decreasing amplitude is first and then smaller, as shown in Figure 7 (a). When the nominal cutter radius is different, the influence of error on the contact area is consistent. And the area of the contact area is sensitive to parallel error, as shown in Figure 7 (b).

\section{Conclusions}

Based on the theoretical research of the existing literature, the influence of the parallelism error of the gear auxiliary axis on the contact ellipse is analyzed. [11]. The following conclusions are drawn:

1) The adaptability of the point contact arc-toothed cylindrical gear to the parallelism installation error is better than the line contact. The gear is better. A gear with a too large radius of the tooth line is very sensitive to the parallel error. Since the spur gear can be regarded as an arc-toothed cylindrical gear with an infinite radius of the tooth line, as long as the appropriate tooth line radius is taken, under the same bearing capacity, the arc the spur gear has better error tolerance than the spur gear.

2) When the installation error is small, the line contact gear pair is selected, and the bearing capacity is large; when the installation error is large, the point contact gear pair is selected, and the comprehensive performance of the bearing capacity and the error adaptability is better. In summary, the arc-toothed cylindrical gear has a larger bearing capacity than the spur gear, and does not require the tooth shaping.

3) Increasing the gear load will increase the amount of elastic deformation, and the corresponding contact ellipse area will also increase, and the point contact will gradually become line contact. Compared with the line contact form, the arc-toothed cylindrical gear and the spur gear have a lower sensitivity to the axial parallelism error.

\section{References}

[1] Liao Yinghua, Liu Gaojun, Sun Xiangguo. Development of an intelligent CAD system for involute cylindrical gear cutting tools [J]. Applied Mechanics and Materials, Mechatronics and Applied Mechanics III, 2014, 532: 249-252.

[2] Dai Fengyan, Shi Zhaoyao, Lin Jiachun. Development and research on gear noise test system [J]. Advanced Materials Research, Engineering Solutions for Manufacturing Processes IV, 2014, 889-890: 718-721.

[3] T. Masuyama et al. Bending strength simulation of asymmetric involute tooth gears [J]. Journal of Advanced Mechanical Design, Systems and Manufacturing, 2015, 9 (5): 15-00485.

[4] Li Haixiang. Basic theory and experimental research on involute arc gear transmission [D]. Chongqing University, 2012.

[5] Sun Xiaowei. Characteristics and processing methods of spiral gears [J]. Mechanical Engineer, 1999 (8): 22-22.

[6] Song Aiping, Wu Weiwei, Gao Shang, Gao Wenjie. Ideal Geometric Parameters of Arc-toothed Cylindrical Gear and Its Processing Method [J]. Journal of Shanghai Jiaotong University, 2010. 44 (12). 1735-1740.

[7] F. L. Litvin. Gear Geometry and Applied Theory [M]. Shanghai: Shanghai Science and Technology Press, 2008.

[8] R. T. Tseng, C. B. Tsay. Contact Characteristics of Cylindrical Gears with Curvilinear Shaped Teeth [J]. Mechanism and Machine Theory, 2004, 39: 905-919.

[9] FL Litvin et al. Topology of Modified Surfaces of Involute Helical Gears with Line Contact Developed for Improvement of Bearing Contact, Reduction of Transmission Errors, and Stress Analysis [J]. Mathematical and Computer Modelling, 2005, 42: 1063-1078.

[10] Tang Jinyuan, Chen Xingming, Luo Caiwang. Contact Analysis of Cylindrical Gear Considering Tooth Shape Modification and Installation Error [J]. Journal of Central South University: Natural Science, 2012, 43 (5).

[11] Jijun Sun, Li Hou, Contact Strength Analysis of Circular-Arc-Tooth-Trace-Cylindrical Gear [J]. Journal of the Brazilian Society of Mechanical Sciences and Engineering, 2016, 38 (3): 999-1005. 